\title{
Scaling theory of three-dimensional spinodal turbulence
}

\author{
V. M. Kendon* \\ Department of Physics and Astronomy, JCMB King's Buildings, University of Edinburgh, Mayfield Road, \\ Edinburgh EH9 3JZ, United Kingdom
}

(Received 19 October 1999)

\begin{abstract}
A scaling theory for spinodal decomposition in the inertial hydrodynamic regime is presented. The scaling involves three relevant length scales, the domain size, the Taylor microscale, and the Kolmogorov dissipation scale. This allows for the presence of an inertial "energy cascade," familiar from theories of turbulence, and improves on earlier scaling treatments based on a single length: these, it is shown, cannot be reconciled with energy conservation. This theory reconciles the $t^{2 / 3}$ scaling of the domain size, predicted by simple scaling, with the physical expectation of a saturating Reynolds number at late times.
\end{abstract}

PACS number(s): $64.75 .+\mathrm{g}$

A binary fluid mixture will undergo phase separation, if the two fluids are mutually repulsive, below some critical temperature. Presented here is a theoretical study of the dynamics of spinodal decomposition in three dimensions, in a $50 / 50$ mixture where the two fluids are incompressible and have the same shear viscosity, $\eta$, and density, $\rho$. Starting from a completely mixed state, quenched to far below the critical temperature, the initial separation is dominated by diffusion until well-defined interfaces form between two interlocking domains of single fluid regions. The ensuing latestage coarsening is then driven by the interfacial tension, resisted by viscosity as the bulk fluid flows so as to flatten the interfaces and enlarge the domain size. The coarsening can be followed through the average domain size, $L$, most commonly measured by the inverse first moment of the spherically averaged structure factor, $S(k)$ of the difference in the concentrations of the two fluids, $L=2 \pi \int S(k) d k / \int k S(k) d k$. Using a simple (single length scale) scaling theory, Siggia [1] predicted that the domain size first shows linear growth, $L \sim t$, in the viscous hydrodynamic regime. Furukawa [2] extended this, predicting a crossover to a slower growth rate of $t^{2 / 3}$ in the inertial hydrodynamic regime. These growth rates have been observed in numerical simulation, see, e.g., [3-7]. Linear growth has been observed experimentally [8].

In what follows, I show that the simple scaling theory is inconsistent with energy conservation in the inertial regime; a minimal alternative is presented, based on three relevant length scales, which allows both force balance and conservation of energy to be maintained at late times. The alternative scaling theory recovers the $L \sim t^{2 / 3}$ scaling, but gives different scalings for other quantities (such as velocity gradients). This allows the physical requirement, recently emphasized by Grant and Elder [9], that the fluid Reynolds number should not diverge in the long time limit, to be reconciled with the $t^{2 / 3}$ scaling.

The scaling approach developed here ignores the anomalous scaling corrections of modern turbulence theory [10]. The analysis of spinodal decomposition presented below is

\footnotetext{
*Present address: Optics Section, The Blackett Laboratory, Imperial College, London, SW7 2BW, United Kingdom.
}

thus an approximate one, but as legitimate as the Kolmogorov theory of homogeneous turbulence. As such, it is likely to be a useful tool for interpreting simulations and experiments on the inertial hydrodynamic regime of spinodal decomposition, and may be the best description available until the (simpler) problem of homogeneous turbulence is fully solved.

The system can be described by the isothermal incompressible Navier-Stokes equation (NSE),

$$
\rho \frac{\partial \mathbf{v}}{\partial t}+\rho(\mathbf{v} \cdot \nabla) \mathbf{v}=\eta \nabla^{2} \mathbf{v}-\nabla \cdot \mathcal{P}
$$

where $\mathbf{v}$ is the fluid velocity. (This can be represented by a single variable regardless of fluid composition since the two fluids have identical properties.) Included in the pressure tensor, $\mathcal{P}$, is the interfacial stress which comes from capillary forces; the excess Laplace pressure in the curved interface drives the bulk fluid away from regions of tight curvature which eventually collapse into narrow "necks" that break, leading to further enlargment of the remaining bulk domains. Using simple scaling arguments $[1,2,11]$, the interfacial force density, $\nabla \cdot \mathcal{P}$, can be approximated by $\sigma / L^{2}$, where $\sigma$ is the interfacial tension between the two fluids. The interface is assumed to remain locally smooth and completely percolated throughout the phase separation process, with constant $\sigma$. This is reasonable provided diffusion is rapid on the scale, $\xi$, of the interfacial width (to maintain local equilibrium on the time scale of the interfacial motion) while being negligible over scales of the order of the domain size so it does not contribute to the domain growth rate. This requires care in simulation work [7], but in real fluids, $L \gg \xi$ can easily be arranged.

The linear scaling is readily obtained from Eq. (1) by neglecting the inertial terms on the left-hand side (lhs) and equating the viscous term to the interfacial one, using $\mathbf{v} \sim \dot{L}$ and $\nabla \sim 1 / L$. When the inertial terms are no longer negligible, reversing this argument and equating the interfacial force to the inertial terms, produces $L \sim t^{2 / 3}$. This assumes that there is only one relevant macroscopic length scale in the system. (Clearly there is also a microscopic length scale in the width of the interface, but it will be assumed, as usual, that this does not affect the macrosopic growth [12].) 
A Reynolds number can be defined [2] by $\operatorname{Re}_{L}=\rho L \dot{L} / \eta$. Clearly for $L \sim t^{2 / 3}, \operatorname{Re}_{L} \sim t^{1 / 3}$, and thus $\operatorname{Re}_{L}$ is predicted to grow without bound in the inertial regime. Recently, Grant and Elder [9] suggested that this unbounded growth of the Reynolds number is unphysical, and there should be a further crossover of the domain growth rate to $t^{1 / 2}$ (or slower) in order that $\mathrm{Re}_{L}$ remains finite. However, the first simulation studies to reliably reach the inertial regime [7] did not find evidence of any final growth rate slower than $t^{2 / 3}$. Below it is argued that $\operatorname{Re}_{L}$ does not quantify the inertial effects which occur in the bulk fluid, which are instead properly characterized by the Reynolds number, $\operatorname{Re}_{\mathbf{v}}=\rho|\mathbf{v} \cdot \nabla \mathbf{v}| /\left(\eta\left|\nabla^{2} \mathbf{v}\right|\right)$, that is, the ratio of the nonlinear to the viscous terms in the NSE, and that while $\operatorname{Re}_{L}$ diverges, $\operatorname{Re}_{\mathbf{v}}$ does not. It is easily seen that $\mathrm{Re}_{\mathbf{v}}$ must remain finite. Infinite $\mathrm{Re}_{\mathbf{v}}$ implies an infinite energy density, while the fluid mixture starts with a finite energy density from the free energy difference between the mixed and separated states.

I start from the energy equation for the system, which (ignoring heat flow) may be written as

$$
\begin{aligned}
\frac{\partial}{\partial t}\left(\rho v^{2} / 2\right)+\mathbf{v} \cdot \nabla\left(\rho v^{2} / 2\right)= & -\eta(\nabla \mathbf{v})^{2}+\mathbf{v} \cdot \nabla \cdot \mathcal{P} \\
& +(\eta / \rho) \nabla^{2}\left(\rho v^{2} / 2\right),
\end{aligned}
$$

where $(\nabla \mathbf{v})^{2} \equiv(\nabla \mathbf{v}):(\nabla \mathbf{v})$. Since we are not concerned with the local convective or diffusive energy flows, it is more convenient to average over the whole system and write the global energy balance per unit volume as

$$
\frac{\mathrm{d}}{\mathrm{d} t}\left\langle\rho v^{2} / 2\right\rangle=-\eta\left\langle(\nabla \mathbf{v})^{2}\right\rangle+\varepsilon_{\mathrm{in}},
$$

where $\varepsilon_{\text {in }}$ is the rate of energy transfer to the fluid from the interface, which can be approximated by $\sigma \dot{L} / L^{2}$ (force $\times$ velocity).

I now show that, in the inertial hydrodynamic regime, the simple scaling theory is inconsistent with energy conservation, by considering the behavior of the global energy balance under the scaling predicted for this regime [2]. Applying the simple scaling to each term in Eq. (3), and replacing $L$ by $t^{2 / 3}$ gives

$$
-\rho t^{-5 / 3} \sim-\eta t^{-2}+\sigma t^{-5 / 3} .
$$

The dissipation term $\left(-\eta t^{-2}\right)$ clearly becomes negligible compared to the other two terms, as originally assumed. However, the kinetic energy in the fluid and the energy stored in the interface are both decreasing over time; thus, energy conservation cannot possibly be maintained except by including the dissipation term in the energy balance equation. Likewise, the viscous term should never be neglected in the NSE because it involves the highest order in derivatives and is thus a singular perturbation: however small $\eta$ is, the asymptotic physics is radically altered from that with $\eta=0$.

I now allow for more general scaling behavior in the NSE and global energy balance equations by introducing two new lengths, $L_{\nabla}$ and $L_{\nabla^{2}}$, with associated scaling exponents $\alpha^{\prime}$ and $\alpha^{\prime \prime}$, for the velocity first and second derivatives respectively, and allowing the velocity to scale as $t^{\beta}$. Terms asso- ciated with the interface are still assumed to have scaling determined by the domain size. The scaling quantities are defined as follows:

$$
\begin{aligned}
\text { domain size: } & L \sim t^{\alpha}, \\
\text { fluid velocity: } & \mathbf{v} \sim t^{\beta}, \\
\text { velocity first derivative: } & \nabla \mathbf{v} \sim \mathbf{v} / L_{\nabla} \sim t^{\beta-\alpha^{\prime}}, \\
\text { velocity second derivative: } & \nabla^{2} \mathbf{v} \sim \mathbf{v} / L_{\nabla^{2}}^{2} \sim t^{\beta-2 \alpha^{\prime \prime}} .
\end{aligned}
$$

This supposes that the interface remains smooth, but allows nontrivial structure in the fluid velocity at smaller scales. Using these scalings to write the NSE, Eq. (1), and energy balance equation, Eq. (3), as powers of $t$ gives, respectively,

$\mathrm{NSE}: \quad \rho \beta t^{\beta-1}+\rho t^{2 \beta-\alpha^{\prime}} \sim \eta t^{\beta-2 \alpha^{\prime \prime}}+\sigma t^{-2 \alpha}$,

energy: $\quad \rho \beta t^{2 \beta-1} \sim-\eta t^{2 \beta-2 \alpha^{\prime}}+\sigma t^{-\alpha-1}$,

where the prefactors have been left in to facilitate identification of the terms. This more general scaling ignores the anomalous scaling corrections of modern turbulence theory [10], but is sufficient to make progress on the problem of spinodal decomposition. The local energy equation, Eq. (2), becomes

$\rho \beta t^{2 \beta-1}+\rho t^{3 \beta-\alpha^{\prime}} \sim-\eta t^{2 \beta-2 \alpha^{\prime}}+\sigma t^{-\alpha-1}+(\eta / \rho) t^{2 \beta-2 \alpha^{\prime \prime}}$.

Solutions for $\alpha, \alpha^{\prime}, \alpha^{\prime \prime}$, and $\beta$ in Eqs. (5) and (6) will later be checked in Eq. (7) to confirm that there are no discrepancies predicted for the behavior of the local energy flows within this three-length-scaling analysis.

As already pointed out, dissipation must remain significant, so we look for a three-way balance between the terms in the energy balance equation, Eq. (6), giving for the exponents,

$$
2 \beta-1=2 \beta-2 \alpha^{\prime}=-\alpha-1 .
$$

This gives $\alpha^{\prime}=1 / 2$ and $\beta=-\alpha / 2$. Substituting these back into the NSE, Eq. (5), gives

$$
\rho t^{-\alpha / 2-1}+\rho t^{-\alpha-1 / 2} \sim \eta t^{-\alpha / 2-2 \alpha^{\prime \prime}}+\sigma t^{-2 \alpha} .
$$

There is no solution to this with all four terms having the same exponent; solutions can instead be found by balancing the terms off in pairs. Numbering the terms $1-4$ from left to right, the pairing that gives the inertial regime scaling is term 1 with term 4 , and term 2 with term 3 , giving $\alpha=2 / 3$, and $\alpha^{\prime \prime}=5 / 12$, with $\beta=-\alpha / 2=-1 / 3$. The four terms in the NSE thus scale as follows:

$$
\rho t^{-4 / 3}+\rho t^{-7 / 6} \sim \eta t^{-7 / 6}+\sigma t^{-4 / 3} .
$$

The physical interpretation of this is that the moving interface gives rise to large scale velocity motion via $\rho \partial \mathbf{v} / \partial t$. The nonlinear term, $\rho \mathbf{v} \cdot \nabla \mathbf{v}$, then transfers the energy from large length scales to small length scales where it is finally removed by dissipation. This is the familiar "energy cascade" of turbulence theory. Note that scaling arguments only pre- 
TABLE I. Summary of predicted scaling exponents for the viscous and inertial regimes. The new theory has the same predictions for the viscous regime as the simple theory, apart from the NSE term $\rho \mathbf{v} \cdot \nabla \mathbf{v}$. Entries are powers of time, $t$; an entry of 0 indicates the quantity is constant, while an entry of $=0$ indicates the quantity is assumed to be zero in the viscous approximation. Bold entries indicate new scaling predictions that differ from the simple theory.

\begin{tabular}{lcrrr}
\hline \hline \multirow{2}{*}{ Quantity } & & Viscous & \multicolumn{2}{c}{ Inertial regime } \\
\cline { 3 - 5 } & & regime & simple scaling & new scaling \\
\hline Domain size & & 1 & $2 / 3$ & $2 / 3$ \\
Length for fluid velocity first derivative & $L_{\nabla}$ & 1 & $2 / 3$ & $\mathbf{1 / 2}$ \\
Length for fluid velocity second derivative & $L_{\nabla^{2}}$ & 1 & $2 / 3$ & $\mathbf{5 / 1 2}$ \\
Fluid velocity & $\mathbf{v}$ & 0 & $-1 / 3$ & $-1 / 3$ \\
& $\rho \partial \mathbf{v} / \partial t$ & $=0$ & $-4 / 3$ & $-4 / 3$ \\
NSE & $\rho \mathbf{v} \cdot \nabla \mathbf{v}$ & $-0 /-\mathbf{1}$ & $-4 / 3$ & $-\mathbf{7 / 6}$ \\
terms & $\eta \nabla^{2} \mathbf{v}$ & -2 & $-5 / 3$ & $-\mathbf{7 / 6}$ \\
& $\sigma / L^{2}$ & -2 & $-4 / 3$ & $-4 / 3$ \\
Reynolds number from interface & $\operatorname{Re}_{L}=(\rho / \eta) L \dot{3}$ & 1 & $1 / 3$ & $1 / 3$ \\
Reynolds number in fluid & $\operatorname{Re}_{\mathbf{v}}=\rho \mathbf{v} \cdot \nabla \mathbf{v} / \eta \nabla^{2} \mathbf{v}$ & $=0$ & $1 / 3$ & $\mathbf{0}$ \\
Dissipation rate & $\varepsilon=\eta(\nabla \mathbf{v})^{2}$ & -2 & -2 & $-\mathbf{5 / 3}$ \\
Taylor microscale & $\lambda=\left(5 \eta\left\langle v^{2}\right\rangle / \varepsilon\right)^{1 / 2}$ & 1 & $2 / 3$ & $\mathbf{1 / 2}$ \\
Kolmogorov dissipation scale & $\lambda_{d}=2 \pi\left(\eta^{3} / \rho^{3} \varepsilon\right)^{1 / 4}$ & $1 / 2$ & $1 / 2$ & $\mathbf{5 / 1 2}$ \\
\hline \hline
\end{tabular}

dict that the paired terms balance approximately, so the nonlinear term, $\rho \mathbf{v} \cdot \nabla \mathbf{v}$, is, in general, larger than the viscous term, $\eta \nabla^{2} \mathbf{v}$, due to the energy "in transit" from large to small scales, contained in a series of eddies of decreasing size. The transverse components of $\rho \partial \mathbf{v} / \partial t$ are thus also larger than required to balance the interfacial force, to account for the rotational motion from $\rho \mathbf{v} \cdot \nabla \mathbf{v}$. The length scales associated with $\nabla$ and $\nabla^{2}$ both grow more slowly than $L$, with $L_{\nabla} \sim t^{1 / 2}$, and $L_{\nabla^{2}} \sim t^{5 / 12}$, so there is an increasing separation of length scales within the system. The dissipation is thus decoupled from the interfacial energy input, and no longer affects the domain growth rate. The Reynolds number, defined as the ratio of the nonlinear to viscous terms, $\operatorname{Re}_{\mathbf{v}}=\rho|\mathbf{v} \cdot \nabla \mathbf{v}| /\left(\eta\left|\nabla^{2} \mathbf{v}\right|\right)$, remains finite (satisfying the physical demand of Grant and Elder [9]), while the domain size grows as $t^{2 / 3}$ (contrary to their deduction that $\alpha \leqslant \frac{1}{2}$ ). With this scaling, the local energy equation, Eq. (7), becomes

$$
-\frac{\rho}{3} t^{-5 / 3}+\rho t^{-3 / 2} \sim-\eta t^{-5 / 3}+\sigma t^{-5 / 3}+\frac{\eta}{\rho} t^{-3 / 2} .
$$

The local convective and diffusive terms are dominant, and balance each other, representing the energy moved around by the turbulent fluid flow.

These results are summarized in Table I, alongside the predictions of the simple scaling theory, for comparison. (Results for the viscous hydrodynamic regime are also shown.) In particular, notice that in the new scaling theory for the inertial regime, the lengths $L_{\nabla}$ and $L_{\nabla^{2}}$ have the same scaling as $\lambda=\left(5 \eta\left\langle v^{2}\right\rangle / \varepsilon\right)^{1 / 2}$, the Taylor microscale, and $\lambda_{d}$ $=2 \pi\left(\eta^{3} / \rho^{3} \varepsilon\right)^{1 / 4}$, the Kolmogorov dissipation scale respectively. The Taylor microscale characterizes the length scales in a turbulent fluid at which dissipation becomes significant, while the Kolmogorov dissipation scale marks the smallscale end of the dissipation range [13]. Thus, although these new scaling results have been obtained without specific input from turbulence theory, the extra length scales for the veloc- ity derivatives turn out to (within prefactors) coincide with key characteristic quantities in turbulence phenomenology, providing strong support for the new theory.

It is easily shown that the familiar linear scaling of the viscous regime [1] is consistent with energy conservation by substituting $\alpha=\alpha^{\prime}=\alpha^{\prime \prime}=1$ and $\beta=0$ into Eqs. (5) and (6), giving

$$
\begin{gathered}
\rho t^{-1} \sim \eta t^{-2}+\sigma t^{-2}, \\
\eta t^{-2} \sim \sigma t^{-2},
\end{gathered}
$$

respectively. In the energy equation, Eq. (13), there is a simple balance between energy input and dissipation, but in the NSE, Eq. (12), the nonlinear term on the lhs, which was assumed to be negligible in the original simple scaling argument, appears to be decaying more slowly $\left(t^{-1}\right)$ than the rhs terms $\left(t^{-2}\right)$. Recalling that the viscous regime is not the long time asymptotic regime, and only expected to hold for times earlier than some crossover time (before which the nonlinear term will be smaller than the other two terms), this apparent difficulty is eliminated.

There are a few further potential solutions to the exponents in Eqs. (5) and (6), which I will now discuss briefly. All can be eliminated on physical grounds. In the inertial regime, where the NSE terms are balanced in pairs, the other possible pairings of terms must checked. It is not possible to balance term 1 with term 2 and term 3 with term 4 because the nonlinear term (term 2) is a force perpendicular to $\mathbf{v}$ (this is obvious when it is written in the alternative form, $-\rho \mathbf{v}$ $\times \nabla \times \mathbf{v})$ and therefore it cannot change the magnitude of $\mathbf{v}$, impying $\beta=0$. However, term 1 is proportional to $\beta$, so is only nonzero if $\beta \neq 0$. Matching term 1 with term 3 , and term 2 with term 4 gives a solution for the exponents of, $\alpha$ $=1 / 2, \alpha^{\prime}=1 / 2, \alpha^{\prime \prime}=1 / 2$ and $\beta=-1 / 4$. Physically, this solution has just one length scale in the system, but the velocity 
is decoupled from the interface, $\mathbf{v} \sim t^{-1 / 4}$, while $\dot{L}(t) \sim t^{-1 / 2}$. In the local energy equation, Eq. (7), this solution becomes

$$
-\frac{\rho}{4} t^{-3 / 2}+\rho t^{-5 / 4} \sim-\eta t^{-3 / 2}+\sigma t^{-3 / 2}+\frac{\eta}{\rho} t^{-3 / 2} .
$$

The convective term, $\rho t^{-5 / 4}$, will eventually come to dominate over all the other terms; thus, it cannot represent an asymptotic solution for late times. There is one further solution for $\beta=0$, obtained by solving Eqs. (5) and (6) for the remaining exponents, $\alpha, \alpha^{\prime}$, and $\alpha^{\prime \prime}$, giving, $\alpha=1 / 3, \alpha^{\prime}$ $=2 / 3$, and $\alpha^{\prime \prime}=1 / 3$. The length scale for velocity gradients is related to the domain size by $L_{\nabla} \sim L^{2}(t)$, suggesting that the nonlinear term is mixing on scales larger than the domain size. Physically, this could correspond to a "turbulent remixing" regime as suggested by Grant and Elder [9]. However, the resulting breakup of the interface is liable to invalidate the assumptions made in deriving the scaling approximation for the interfacial force. Thus, although this appears to be a consistent solution to the NSE and energy balance equations, it seems unlikely that the system could, in fact, ever achieve such a scaling.

In summary, I have obtained consistent scaling behavior for domain growth in the spinodal decomposition of a symmetric binary fluid mixture by including two extra macroscopic length scales for the velocity derivatives to enable energy balance to be satisfied. The velocity itself is still found to scale as $\dot{L}(T)$. In a system restricted to derivatives up to second order (the NSE is, itself, an approximation based on separation of macroscopic and molecular length and time scales), one length scale per derivative would seem to be a reasonable maximum for the purpose of simple scaling arguments, and it does not seem to be possible to satisfy the NSE and energy equations using fewer.

With this new scaling, the inertial regime scaling of $L$ $\sim t^{2 / 3}$ is maintained while the fluid Reynolds number, $\operatorname{Re}_{\mathbf{v}}$, measured as the actual ratio of the nonlinear to the viscous terms in the NSE, remains finite. The Reynolds number obtained from the domain size, $\mathrm{Re}_{L}$, is not a good estimate of $\mathrm{Re}_{\mathbf{v}}$ in the inertial regime so it is not physically significant that $\operatorname{Re}_{L}$ continues to grow without bound. The key point for the scaling behavior of $L$ is that the driving force from the interface balances against the acceleration term alone, so is decoupled from what happens in the remainder of the fluid motion. The nonlinear and viscous terms are free to find their own balance independent of the driving force, provided that they do, eventually, remove energy from the system.

There is, as yet, no simulation or experimental work that tests this new scaling theory. The simulation work reported in $[7,14]$, while showing hints of possible different scaling for the NSE terms, was not able to probe far enough into the inertial regime to provide a significant test. Using $L_{0}$ $=\eta^{2} /(\rho \sigma)$ and $t_{0}=\eta^{3} /\left(\rho \sigma^{2}\right)$ to provide nondimensional length $\left(L / L_{0}\right)$ and time $\left(t / t_{0}\right)$, Ref. [7] suggests that $L / L_{0}$ $>10^{6}, t / t_{0}>10^{9}$ will be required, at least an order of magnitude beyond present simulation capabilities. Experimental work, though more feasible, will not be easy to perform. Using values for water, $\eta=10^{-3} \mathrm{Kg} \mathrm{m}^{-1} \mathrm{~s}^{-1}$, and for waterparaffin, $\sigma=2.4 \times 10^{-2} \mathrm{Kg} \mathrm{s}^{-2}$, with the projection from simulation work of $L / L_{0}>10^{6}$ to estimate the interfacial force $\left(\sigma / L^{2}\right)$, the density matching of the two mutually repulsive fluids must be better than one part in $10^{4}$ for terrestrial gravitational effects to remain small in the required regime $(L \sim 4 \mathrm{~cm})$. The micro-gravity environment of the space shuttle would ease this constraint [15].

I would like to thank Mike Cates and Ignacio Pagonabarraga for valuable discussions and a careful reading of the manuscript, and EPSRC for financial support. This work was funded in part by EPSRC GR/M56234.
[1] E.D. Siggia, Phys. Rev. A 20, 595 (1979).

[2] H. Furukawa, Phys. Rev. A 31, 1103 (1985).

[3] C. Appert, J.F. Olson, D.H. Rothman, and S. Zaleski, J. Stat. Phys. 81, 181 (1995).

[4] M. Laradji, S. Toxvaerd, and O.G. Mouritsen, Phys. Rev. Lett. 77, 2253 (1996).

[5] S. Bastea and J.L. Lebowitz, Phys. Rev. Lett. 78, 3499 (1997).

[6] S.I. Jury, P. Bladon, S. Krishna, and M.E. Cates, Phys. Rev. E 59, R2535 (1999).

[7] V.M. Kendon, J.-C. Desplat, P. Bladon, and M.E. Cates, Phys. Rev. Lett. 83, 576 (1999).

[8] See, for example, K. Kubota, N. Kuwahara, H. Eda, and M. Sakazume, Phys. Rev. A 45, R3377 (1992); S. Chen, G. Doolen, R. Kraichnan, and Z.-S. She, Phys. Fluids A 5, 458 (1993); T. Hashimoto, H. Jinnai, H. Hasegawa, and C.C. Han, Physica A 204, 261 (1994).

[9] M. Grant and K.R. Elder, Phys. Rev. Lett. 81, 14 (1999).
[10] See for example, U. Frisch, Turbulence: The Legacy of A. N. Kolmogorov (CUP, Cambridge, England, 1995).

[11] A.J. Bray, Adv. Phys. 43, 357 (1994).

[12] Studies of droplets breaking [J. Eggers, Phys. Fluids 7, 941 (1995)] and of the anomalous scaling in turbulence [G. Stolovitzky, K.R. Sreenivasan, and A. Juneja, Phys. Rev. E 48, R3217 (1993)] support the view that microscopic length scales do not influence macroscopic behavior when considering the NSE. (But see [6].)

[13] See, for example, A.S. Monin and A.M. Yaglom, Statistical Fluid Mechanics, edited by J. Lumley (MIT Press, Cambridge, MA, 1975).

[14] V.M. Kendon, M.E. Cates, J.-C. Desplat, I. Pagonabarraga, and P. Bladon (unpublished).

[15] G. Cambon, B. Zappoli, R. Marcout, Y. Garrabos, F. Cansell, D. Beysens, B. Billia, and H. Jamgotchian, Acta Astron. 41, 447 (1997). 